Proceedings

\title{
Ultrasonic Measurement of Velocity Profile on Bubbly Flow Using a Single Resonant Frequency ${ }^{\dagger}$
}

\author{
Wongsakorn Wongsaroj ${ }^{1, *}$, Ari Hamdani ${ }^{2}$, Natee Thong-un ${ }^{3}$, Hideharu Takahashi ${ }^{2}$ and \\ Hiroshige Kikura ${ }^{2}$ \\ 1 Department of Mechanical Engineering, Tokyo Institute of Technology, Tokyo 152-8550, Japan \\ 2 Laboratory for Advanced Nuclear Energy, Institute of Innovative Research, Tokyo Institute of Technology, \\ Tokyo 152-8550, Japan; hamdani.a.aa@m.titech.ac.jp (A.H.); htakahashi@lane.iir.titech.ac.jp (H.T.); \\ kikura@lane.iir.titech.ac.jp (H.K.) \\ 3 Department of Instrumentation and Electronics Engineering, King Mongkut's University of Technology \\ North Bangkok, Bangkok 10800, Thailand; natee.t@eng.kmutnb.ac.th \\ * Correspondence: wongsaroj.w.aa@m.titech.ac.jp; Tel.: +81-(0)3-5734-2959 \\ + Presented at the 18th International Conference on Experimental Mechanics (ICEM18), Brussels, Belgium, \\ 1-5 July 2018.
}

Published: 16 July 2018

\begin{abstract}
The present paper describes a measurement technique for phase-separated velocity profile measurements in the two-phase bubbly flow. The Ultrasonic Velocity Profiler (UVP) method which is nonintrusive measurement, is applied to obtain an instantaneous velocity profile of liquid and bubble separately by using only one resonant frequency. To achieve this target, developed algorithm, which can decompose frequency component of the Doppler signal affected by liquid and bubble, is applied in the UVP system to obtain and separate instantaneous velocity profile of both phases. For confirming the applicability of modified measurement system, the developed UVP was used for the measurement of the velocity profile in bubbly flow on vertical pipe flow apparatus, the measurement accuracy was validated by UVP Original and Particle Image Velocimetry (PIV) method. Finally, the UVP was applied to experiment for observing velocity distribution of both phases in a bubble column.
\end{abstract}

Keywords: ultrasonic; velocity profile; bubbly flow; single resonant frequency

\section{Introduction}

Two-phase bubbly flow is a fundamental phenomenon which occurs in many industrial processes such as thermal power plant, chemical processes, nuclear reactors, and so on. It has been investigated continuously over several decades. However, due to its complexity, some characteristics of the phenomenon have not yet been understood. The velocity profile of liquid and bubble is an important parameter in the bubbly flow. Also, it is used to calculate other parameters such as void fraction and slip velocity. Many intrusive techniques have been employed to investigate velocity distribution in bubbly flow such as conductive probe [1]. This technique disturbs the flow and leads to lifetime distortion. Therefore, nonintrusive measurement technique was proposed, such as Particle Image Velocimetry (PIV) [2]. This technique has been utilized to measure the rising velocity of liquid (particles) and bubbles. However, the method requires a transparent test section and obviously fail if the working fluid is opaque.

The Ultrasonic Velocity Profiler (UVP) method is a nonintrusive measurement, which can measure the velocity profile of the fluid even though is opaque. The method does not require the transparent test section. The UVP measurement uses a pulsed echography of an ultrasonic wave reflected from moving reflector such as particle dispersed in a fluid to obtain an instantaneous 
velocity profile of the fluid along its measurement line. Takeda [3] was the first of proposing the UVP method to measure the velocity profile of liquid. Then, Aritomi et al. [4] developed a system, which combined the UVP system and video data processing unit to inspect the velocity distributions of each phase in the bubbly flow. The measurement technique was based on statistic method. The velocity of both phases could be obtained separately. The limitation of separation occurs when the velocity of both phases is not much different. Suzuki et al. [5] applied the pattern recognition of the velocity profile in UVP measurement to distinguish a phase of the velocity profile. However, the technique could be only applied for limited flow condition. Murakawa et al. [6] employed multi-waves TDX transducer and time domain cross-correlation method (UTDC) to measure and separate liquid and bubble velocity profile with pattern recognition of ultrasonic echo amplitude. Although this technique could obtain the instantaneous velocity of both phases, more devices were required owing to two resonant frequencies were needed. Therefore, the measurement system is more complex.

This work proposes a developed technique of UVP system for obtaining instantaneous velocity profile of bubbly flow using single resonant frequency. Hence, the equipment of the measurement system is minimized because the system employs only a single resonant frequency transducer, single channel pulser/receiver, and simple processing equipment. The velocity profile of both phases can be obtained and classified even though the velocity data of liquid and bubble occurs on the same measurement channel. Also, the velocity of liquid and bubbles can be distinguished, although similarities are observed in each phase. To confirm the applicability of developed UVP, the experiment was conducted on a vertical pipe flow apparatus, measurement accuracy was guaranteed by comparing with UVP Original and PIV method. Moreover, the UVP is applied to experiment in a bubble column for observing velocity distribution of both phases.

\section{Measurement Technique}

\subsection{The Principle of Ultrasonic Velocity Profiler (UVP)}

UVP [3] is a method to obtain instantaneous velocity profiles of fluid using ultrasonic waves by means of an ultrasound reflector. Figure 1 illustrates the UVP principle consisting of ultrasound transmission and the echo signal. An ultrasonic pulse is emitted from the transducer along the measurement line repeatedly, and the echo reflected from the surface of the reflector such as small particle is derived from the same transducer. The particles dispersed in the fluid according to the main flow.

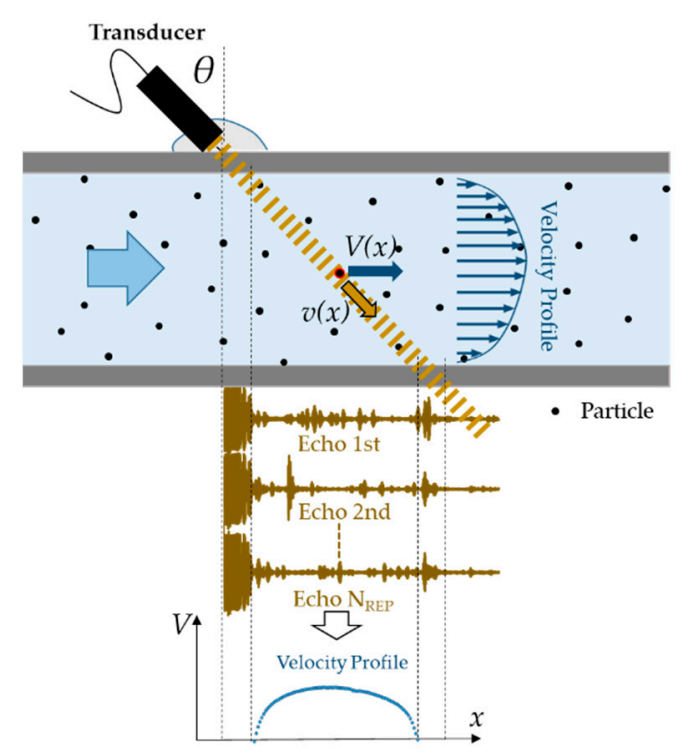

Figure 1. UVP measurement configuration, echo signal and velocity profile reconstruction. 
In the case of water, the density of the particle is nearly equal to water $\left(0.98 \mathrm{~g} / \mathrm{cm}^{3}\right)$. Therefore, the velocity of a particle is assumed to be equal to the velocity of water. The echo signals reflected from moving particles contain Doppler signal. The Doppler frequency $f_{D}(x)$ directly relates to a velocity of moving particle. Consequently, the velocity of the particle at that position $V(x)$ can be obtained as following Equation (1). Therefore, the velocity profile along channel diameter can be computed if tracer particles are sufficiently dispersed.

$$
V(x)=\frac{c f_{D}(x)}{2 f_{0} \sin \theta}
$$

where $f_{0}$ is the basic frequency of the ultrasonic wave, $\theta$ is the incident angle and $x$ is a position or measurement channel.

\subsection{UVP Measurement in Bubbly Flow with Single Resonant Frequency}

In bubbly flow, Doppler signal is demodulated from echo signals reflected by particle (liquid) and bubble. Its frequency and amplitude inform the velocity and the identity of each reflector respectively. In some case, the position of particle and bubble occurs in the same measurement channels in the period of the number of repetition (NREP). Hence, it is difficult to distinguish velocity in both phases. When this behavior occurs, multi-frequency and difference of amplitude in the Doppler signal are generated as shown in Figure 2. Therefore, advanced signal processing is required to analyze the effect of Doppler signal for decomposing Doppler frequency of particle and bubble. Then, the velocity of both phases can be classified.

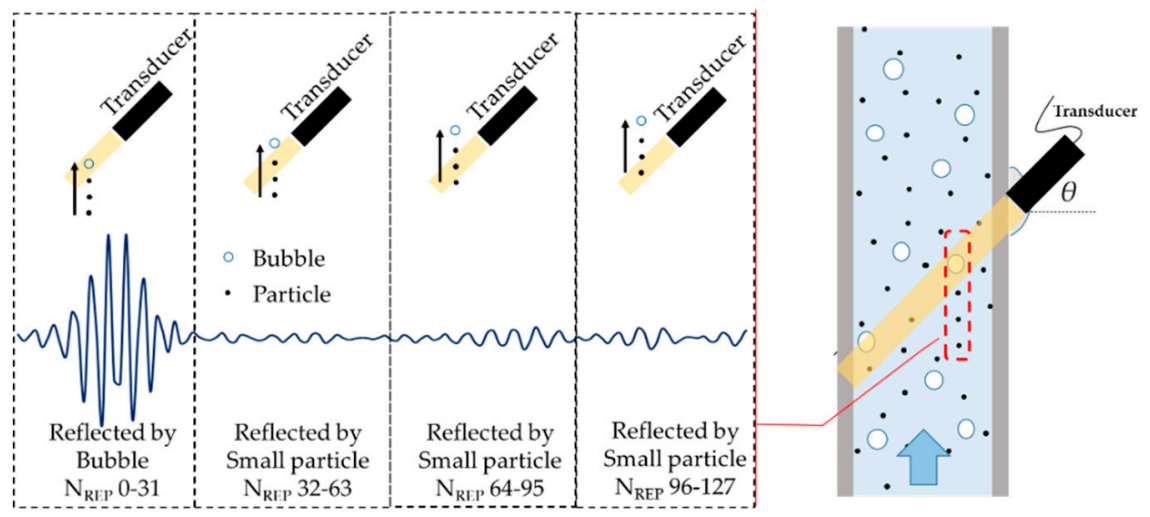

Figure 2. Doppler signal in one measurement channel on the bubbly flow.

The integration of time-frequency analysis and Doppler amplitude classification is proposed for this case as shown in Figure 3a. Short-Time Fourier Transform (STFT) [7] is selected to be a timefrequency estimator. It decomposes frequency components of the Doppler signal according to the time. The frequency value can be observed from a peak energy occurring on spectrogram in each time location as express in Equations (2) and (3).

$$
\begin{aligned}
X\left(k, f_{D}\right)= & \sum_{n=0}^{N_{\text {REP }}-1} D(n) W_{n}\left(n-k S_{n}\right) \exp \left(-j n 2 \pi f_{D}\right) \\
& P\left(k, f_{D}\right)=\left|X\left(k, f_{D}\right)\right|^{2}
\end{aligned}
$$

where $k$ is discrete time, $D(n)$ is Doppler signal, $W_{n}$ is window length, $S_{n}$ is time step and $P\left(k, f_{D}\right)$ represents the energy density of spectra in time-frequency function.

Then, each frequency component is classified to be Doppler frequency of particle and bubble by comparing the value of Doppler amplitude in that time location with a threshold value. If the amplitude value is higher than the threshold, the frequency value will be defined as Doppler frequency of bubble. In contrary, if the value is lower than the threshold, the frequency value in that location will be Doppler frequency of particle. The threshold value is based on the statistical data of 
Doppler amplitude reflected by particles and bubbles of different sizes (the Doppler amplitude of the bubble is bigger than a particle because reflection ratio index is higher [8]). The technique is called "Doppler Frequency Decomposition" (DFD). It is applied in UVP measurement system.

Figure $3 \mathrm{~b}$ represents an example of the Doppler frequency classification of DFD technique in one measurement channel, showing the processing system on single-phase flow. The Doppler frequency in each component observed from the peak on the spectrogram is only influenced by particle because the Doppler amplitude of each time location that the peak on the spectrogram occurs is lower than the threshold value. Figure $3 \mathrm{c}$ shows the example in bubbly flow conditions. The frequency data observed from the peak on the spectrogram at point $a, b$, and $c$ is defined as the Doppler frequency of a particle due to Doppler amplitude at the time location is lower than the threshold value. On the contrary, at point $\mathrm{d}$, the Doppler frequency belongs to a bubble because Doppler amplitude is higher than the threshold value.

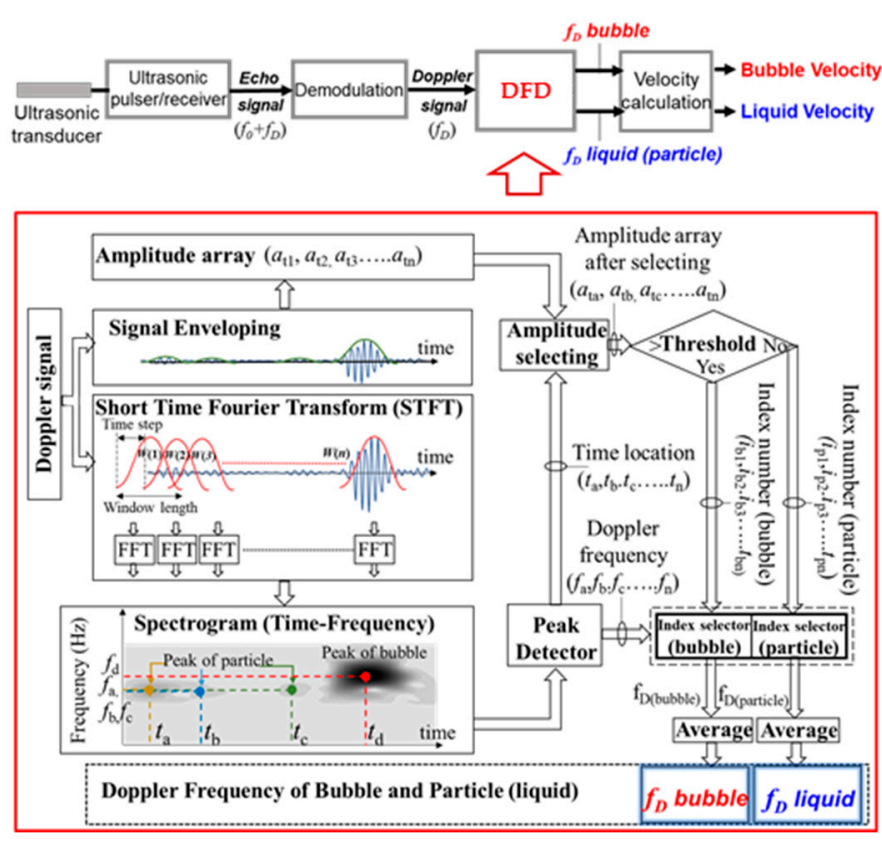

(a)

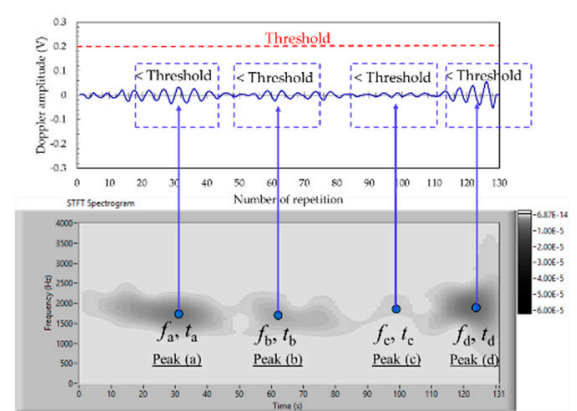

(b)

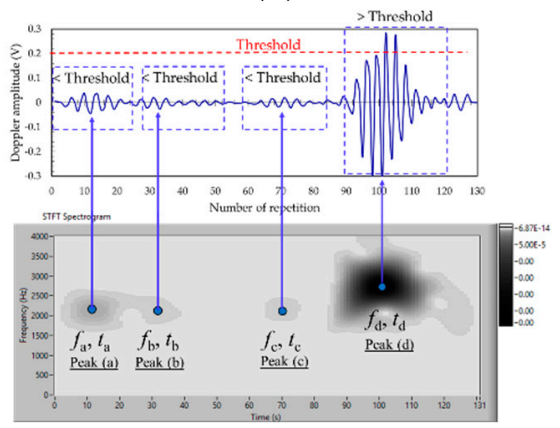

(c)

Figure 3. Doppler frequency classification technique (DFD); (a) function block diagram, (b) example of processing in single-phase flow (only particle), (c) example of processing in bubbly flow (with particle and bubble).

\section{Experimental Results and Discussion}

\subsection{Velocity Profile Measurement of Single Phase and Bubbly Flow in the Pipe}

In this section, the performance of the UVP measurement with DFD technique (UVP-DFD) is validated by the experiment. The experiment was performed on the acrylic vertical pipe by applying a transducer on the outer pipe with an incident angle of $45^{\circ}$. The experimental setup is shown in Figure 4a. A measurement system consisted of the UVP and a PIV system. Working fluid was tap water which was dispersed by nylon particle $80 \mu \mathrm{m}$. Its temperature was controlled around $20 \pm 2$ ${ }^{\circ} \mathrm{C}$. In the test section, the inner diameter of the acrylic pipe was $20 \mathrm{~mm}$ and thickness was $1 \mathrm{~mm}$. It locates downstream from a bubble generator with a distance of 50D. The UVP system consists of a 4 $\mathrm{MHz}$ ultrasonic transducer (imasonic), a Pulser/Receiver (UVP-DUO, Metflow, Lausanne, Switzerland), an 8-bit Digitizer (NI USB 5133, National Instruments Corp, TX, USA) which the sampling speed is $100 \mathrm{MS} / \mathrm{s}$ and a personal computer with LabVIEW program. 
Figure $4 \mathrm{~b}$ shows a result of average velocity profile (5000 instantaneous profile) measurement in single phase liquid flow at the superficial liquid velocity $U_{L} 200 \mathrm{~m} / \mathrm{s}$. The horizontal axis indicates the distance from the wall (r) nominalized by the pipe radius ( $R$ ). The velocity distributions measured by UVP-DFD, UVP Original, and PIV, had almost agreement except near the vicinity of the wall. The small fluctuation occurs because there was the resonance of the overlapped regions between the ultrasonic wave and the pipe wall.

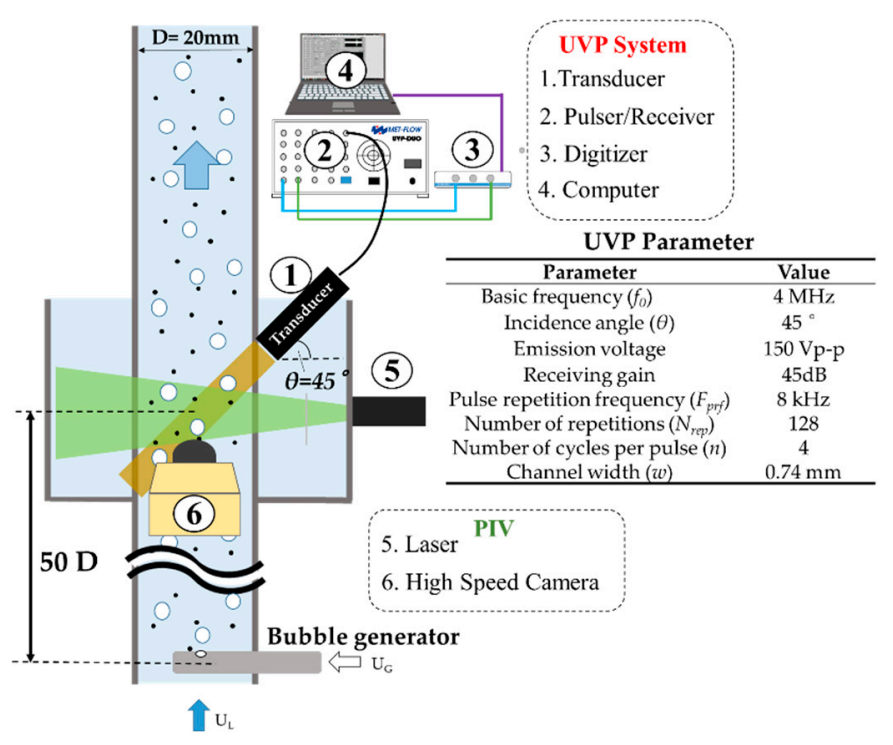

(a)

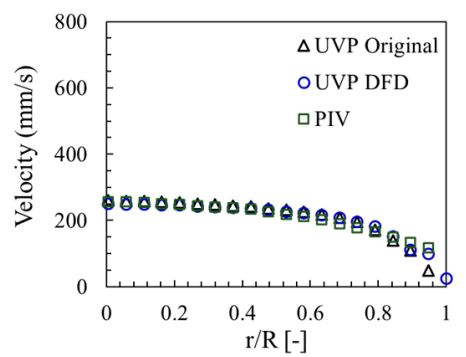

(b)

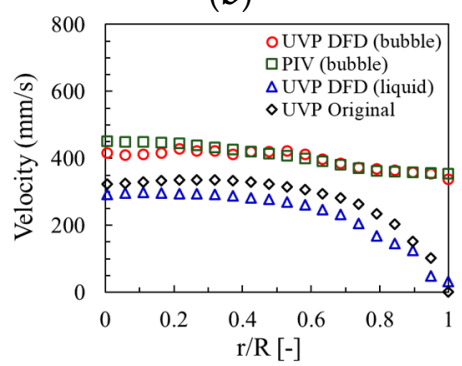

(c)

Figure 4. Measurement of single phase and bubbly flow in the vertical pipe; (a) Experimental apparatus, (b) Average velocity profile at $U_{L}=200 \mathrm{~mm} / \mathrm{s}$ on single phase flow, (c) Average velocity profile at $U_{L}=200 \mathrm{~mm} / \mathrm{s}$ on bubbly flow.

In bubbly flow, the experiment was conducted at $U_{L}=200 \mathrm{~mm} / \mathrm{s}$ and $U_{G}=5.3 \mathrm{~mm} / \mathrm{s}$. The average bubbles diameter in this experiment was about $3 \mathrm{~mm}$. Figure $4 \mathrm{c}$ shows the measurement result of the bubbly flow. The graph shows the average data of velocity profiles. Liquid velocity distribution is the mean of 5000 profile and bubble rising velocity is averaged by a number of data obtained. The result of bubble velocity distribution after separation of UVP-DFD technique was verified with the PIV method. The velocity profiles measured by both methods are almost coincident with each other. The differences were an error of the three-dimensional motion of bubble. Liquid velocity profile after separation was obtained and compared with UVP Original. As a result, the velocity profile of UVP Original was higher than that of UVP-DFD obviously because the influence of bubble motion was not rejected from the profile and slip ratio between the bubble and liquid velocity that occurred. Hence, liquid velocity profile measurement of UVP-DFD in bubbly flow can be confidently trusted owing to delete the bubble velocity data. It can be concluded that UVP-DFD has the applicability to measure velocity profile on single phase and bubbly flow in the pipe since the good agreement of the measurement result with UVP Original and PIV method was obtained apparently.

\subsection{Velocity Profile Measurement of Bubbly Flow in a Bubble Column}

In this section, The UVP-DFD was applied to measure the velocity distribution of the bubbly flow in the bubble column. Working fluid was tap water which dispersed with nylon particle $80 \mu \mathrm{m}$ and bubble. The air at flow rate of $0.6 \mathrm{~L} / \mathrm{min}$ was supplied to the bubble generator for generating the bubble. The motion of the bubble rises upward. It influences to the liquid motion. The experimental setup is shown in Figure 5a. The equipment and parameter setting of UVP were similar to the previous experiment. The transducer was immersed in the water and installed at an incident angle of $45^{\circ}$. The measurement was performed in two different positions; $230 \mathrm{~mm}$ and $100 \mathrm{~mm}$ from the bottom of the tank. Figure $5 \mathrm{~b}$ shows the average data of 5000 instantaneous velocity profile 
measurement. The horizontal axis indicates the distance from the transducer surface. At both positions, the velocity profile of liquid and bubble could be obtained separately. Furthermore, the region of the velocity distribution of both phases could be observed. The result of both positions is reasonable, bubble area at position 1 is wider than at position 2 because the expansion of bubble area happens according to a vertical level of the water in a bubble column. This behavior is also influent to expanding into liquid velocity area. It can be summarized that the UVP-DFD is not only to obtain and separate velocity distribution of both phases but also it can be used to observe the region of the bubble and liquid motion.

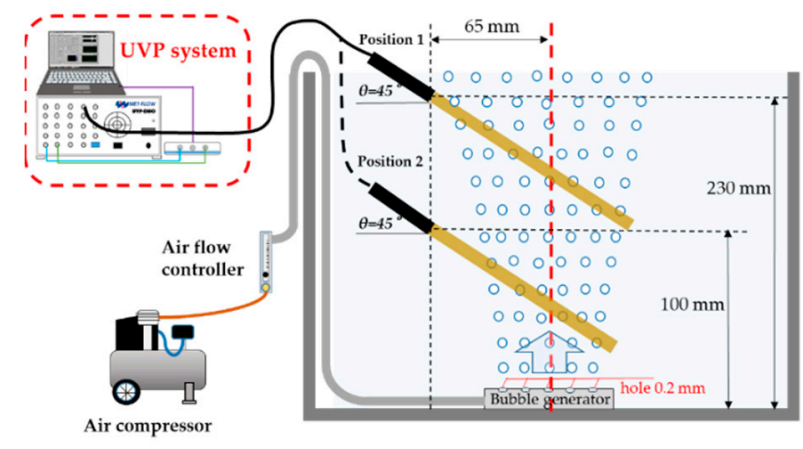

(a)
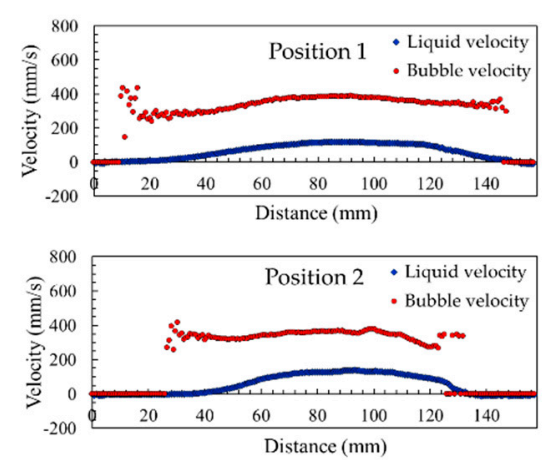

(b)

Figure 5. Velocity profile measurement in bubble column; (a) Experimental apparatus, (b) Average velocity profile.

\section{Conclusions}

The UVP method with single resonant frequency was developed for measuring instantaneous velocity profiles of liquid and bubbles in the two-phase bubbly flow. The system employed only a single resonant frequency transducer, single channel pulser-receiver, and simple data processing unit. Therefore, extra equipment was not needed. The signal processing of conventional UVP was modified by applying Doppler frequency decomposition technique (DFD). It could classify Doppler frequency of particle (liquid phase) and bubble. Hence, the velocity profile of the liquid and bubble could be measured and separated clearly. The experiment was conducted on a vertical pipe flow apparatus. The measurement accuracy of developed UVP (UVP-DFD) was guaranteed due to the result had good agreement with UVP Original and PIV method. Furthermore, the UVP was applicable to observe velocity distribution of both phases in a bubble column.

\section{References}

1. Wu, Q.; Ishii, M. Sensitivity study on double-sensor conductivity probe for the measurement of interfacial area concentration in bubbly flow. Int. J. Multiph. Flow 1999, 25, 155-173.

2. Chen, R.C.; Fan, L.S. Particle image velocimetry for characterizing the flow structure in three-dimensional gas-liquid-solid fluidized beds. Chem. Eng. Sci. 1992, 47, 3615-3622.

3. Takeda, Y. Velocity profile measurement by ultrasonic Doppler shift method. Int. J. Heat Fluid Flow 2008, 7, 313-318.

4. Aritomi, M.; Zhou, S.; Nakajima, M.; Takeda, Y.; Mori, M.; Yoshioka, Y. Measurement system of bubbly flow using ultrasonic velocity profile monitor and video data processing unit. J. Nucl. Sci. Technol. 1996, 3312, 915-923.

5. Suzuki, Y.; Nakagawa, M.; Aritomi, M.; Murakawa, H.; Kikura, H.; Mori, M. Microstructure of the flow field around a bubble in counter-current bubbly flow. Exp. Therm. Fluid Sci. 2002, 26, 221-227.

6. Murakawa, H.; Kikura, H.; Aritomi, M. Application of ultrasonic multi-wave method for two-phase bubbly and slug flow. Flow Meas. Instrum. J. 2008, 19, 205-213. 
7. Sharma, G.K.; Kumar, A.; Rao, C.B.; Jayakumar, T.; Raj, B. Short time Fourier transform analysis for understanding frequency dependent attenuation in austenitic stainless steel. NDTEE Int. J. 2013, 53, 1-7.

8. Murai, Y.; Tasaka, Y.; Nambu, Y.; Takeda, Y.; Gonzalez, S.R. Ultrasonic detection of moving interfaces in gas-liquid two-phase flow. Flow Meas. Instrum. J. 2010, 21, 356-366.

(c)

(C) 2018 by the authors. Licensee MDPI, Basel, Switzerland. This article is an open access article distributed under the terms and conditions of the Creative Commons Attribution (CC BY) license (http://creativecommons.org/licenses/by/4.0/). 